\title{
Angst, Schmerz und Stress - eine unheilige Trias
}

\author{
Christel Nigg \\ Klinik Susenberg, Zürich
}

Der Beitrag von N. Egloff et al. [1] in dieser Ausgabe setzt sich mit den in der täglichen Praxis sehr häufig auftretenden Phänomenen Angst und Schmerz auseinander. Die Darstellung der beiden Empfindungen als einerseits Erleben einer möglichen Bedrohung der körperlichen Integrität und andererseits einer drohenden situativen Gefährdung ist interessant und zeigt, dass es sinnvolle Warnsysteme gibt, die unsere Existenz sichern helfen.

Allerdings können sich diese Phänomene auch zu einer eigenen Krankheitsentität entwickeln, was sehr häufig der Fall ist. Das Konzept der übergreifenden Sensibilisierung durch Stress macht deutlich, wie eng diese beiden Phänomene miteinander verbunden sind. Wichtig ist, dass es eine umfassende Betrachtung dieser Empfindungen braucht, um Behandlungsartefakte zu vermeiden.

Das erste Patientenbeispiel verdeutlicht sehr anschaulich, dass es natürlich bei bedrohlichen körperlichen Erkrankungen wie z.B. einem akuten Koronarsyndrom durch den ischämisch bedingten Schmerz zu massiver Angst und vegetativen Reaktionen durch den ausgelösten Stress kommt, auch wenn die/der Betroffene im Vorfeld seelisch völlig stabil war. Im zweiten Beispiel wird illustriert, wie jahrelanger Stress zu chronischen Schmerzen und im Verlauf auch zu Angst- und Panikstörungen beiträgt. Das dritte Beispiel belegt den Zusammenhang von Stress und Angst mit traumatischen Erfahrungen.

Die Kommentare zu den drei Patientenbeispielen sind mit zahlreichen Studien belegt. Sehr hilfreich in der Kommunikation mit betroffenen Patientinnen und Patienten ist die Erläuterung der gemeinsamen neurophysiologischen Basis, nämlich die überlappende neurochemische Basis beider Systeme und das Vorhandensein von strukturellen Schnittstellen für die Signale. Diese Erläuterungen helfen vor allem denjenigen, die sich gegen eine einseitige
Psychologisierung ihrer Beschwerden wehren, helfen aber auch dem Therapeuten, fortgesetzt überflüssige medizinisch-technische Abklärungen zu vermeiden. Die Betrachtungsweise legt nahe, dass das Behandlungskonzept auf diese Komplexität ausgerichtet sein muss. Weder können rein schmerzstillende Medikamente noch ausschliesslich Psychopharmaka eingesetzt werden, sondern das zugrundeliegende Störungsmuster sollte ganzheitlich therapeutisch angegangen werden. Vor allem die Gewalterfahrung muss sorgfältig und behutsam exploriert werden, sie kann teilweise lange zurückliegen. Gerade in der Grundversorgung sind solche Krankheitsbilder häufig. Es braucht Geduld und die Bereitschaft, diese Patientinnen und Patienten mit Empathie zu betreuen, auch wenn der Erfolg manches Mal auf sich warten lässt. Egloff et al. [1] zeigen exzellent auf, wie wichtig eine klare Vorstellung der zugrundeliegenden Probleme und ein strukturiertes Therapiekonzept der Behandler ist. Henne oder Ei zuerst manchmal nicht ganz einfach zu verstehen.

\section{Bibliografie}

1. Egloff N, Mueller DB, Orlof AM, grosse Holtforth M, Blaettler LT: Die Kopplung von Schmerz, Angst und Stress. Praxis. 2021;110: 263-267.

KD Dr. med. Christel Nigg

Chefärztin, Vorsitzende der Geschäftsleitung

Klinik Susenberg

Schreberweg 9

8044 Zürich

c.nigg@susenbergklinik.ch 